Western University

Scholarship@Western

Business Publications

Business (Richard Ivey School of Business)

2011

\title{
Approches stratégiques des émissions CO2: Les cas de l'industrie cimentière et de l'industrie chimique
}

Diane-Laure Arjaliès

Ivey Business School, darjalies@ivey.ca

Goubet Cécile

Ecole Polytechnique de Palaiseau

Jean-Pierre Ponssard

Ecole Polytechnique de Palaiseau

Follow this and additional works at: https://ir.lib.uwo.ca/iveypub

Part of the Business Administration, Management, and Operations Commons, and the Business Law, Public Responsibility, and Ethics Commons

Citation of this paper:

Arjaliès, D-L., Goubet C. \& Ponssard, J-P. (2011). Approches stratégiques des émissions CO2: Les cas de l'industrie cimentière et de l'industrie chimique, Revue Française de Gestion, 2011, 37(215): 123-146. 


\title{
Approches stratégiques des émissions $\mathrm{CO}_{2}$ :
}

Figures libres ou figures imposées?

\section{Les cas de l'industrie cimentière et de l'industrie chimique}

$$
15 \text { Avril } 2011
$$

Version révisée

Diane Laure Arjaliès ${ }^{1}$, Cécile Goubet ${ }^{2}$ et Jean-Pierre Ponssard ${ }^{3}$

Proposition d'article pour le numéro spécial:

\section{« Le management responsable: de la gestion des risques à l'innovation stratégique "}

\section{Rédacteur invité}

Jean-Claude Dupuis, ESDES-UCLy-Université de Lyon

\begin{abstract}
Résumé
La capacité des entreprises à transformer une contrainte environnementale en source d'opportunité stratégique est un sujet controversé dans la littérature. S’appuyant sur une étude comparative des stratégies de lutte contre les émissions $\mathrm{CO}_{2}$ mises en place par les industries cimentière et chimique, l'article démontre que la latitude des entreprises à adopter une approche proactive face au développement durable est fortement contrainte par les caractéristiques du secteur en termes de dépendance vis-à-vis des ressources naturelles, de flexibilité dans la composition du portefeuille d'activités et de structure du secteur aval.
\end{abstract}

Mots-clefs : $\mathrm{CO}_{2}-$ Développement Durable - Stratégie des firmes - Innovation

*Nous remercions les chaires Business Economics et Finance Durable et Investissement Responsable de l'Ecole Polytechnique pour le soutien financier apporté à ce projet de recherche.

${ }^{1}$ Auteur de correspondance: Département Comptabilité-Contrôle de Gestion, HEC Paris, 1 rue de la Libération 78351 - Jouy-en-Josas, arjalies@hec.fr.

${ }^{2}$ CDC Climat Recherche, 47, rue de la Victoire, 75009 Paris, cecile.goubet@cdcclimat.com.

${ }^{3}$ Département d'Economie, Ecole Polytechnique, 91128 Palaiseau Cedex, Jean

Pierre.Ponssard@polytechnique.edu. 


\section{Introduction}

Au cours de la dernière décennie, le débat ayant trait au réchauffement climatique a considérablement évolué. L’une des principales causes du réchauffement climatique provient de l'accroissement des émissions de gaz à effet de serre (GES) d'origine anthropique. Les entreprises sont doublement concernées: en tant qu'émettrices de GES et en tant qu'actrices du développement de nouveaux modèles de gestion dans lesquels croissance économique et émissions de GES seront déconnectés (Mc Kinsey Global Institute 2008). Depuis le sommet de Rio en 1992, de nombreux Etats se sont engagés à lutter contre le changement climatique et une panoplie d'instruments réglementaires et incitatifs s'est progressivement mise en place. Dans le cadre du protocole de Kyoto, l'Union Européenne a ainsi choisi de répartir son objectif de -8\% de réductions d'émissions de GES entre ses différents Etats membres. Pour atteindre cet objectif, le système d'échange de quotas de l'Union Européenne (EU-ETS), qui couvre les émissions de GES des installations industrielles et électriques, a été mis en place en 2005. La troisième phase de l'EU-ETS concernant la période 2013-2020 fait maintenant état d'un objectif de -21\% par rapport à 2005 .

Anticipant sur le développement de ces réglementations, certaines entreprises se sont unilatéralement engagées dans des actions stratégiques visant à réduire les impacts environnementaux de leurs activités. On a vu par exemple se développer des accords de branche, tels que ceux coordonnés par le $\mathrm{WBCSD}^{4}$ pour l'acier, le ciment, ou ceux pilotés par des associations professionnelles comme dans la chimie. Ces démarches volontaires sont souvent analysées par les économistes comme des tentatives de préemption de la réglementation (Morgensten et Pizer 2007). Pourtant, alors que la plupart des réglementations sont maintenant en place, ces approches volontaires perdurent: au-delà de son aspect réglementaire, le

\footnotetext{
${ }^{4}$ World Business Council for Sustainable Development
} 
changement climatique serait donc devenu pour certaines entreprises un enjeu stratégique. D’ailleurs, nombre d'entreprises développent leur propre vision en la matière en cherchant à en faire un axe de différenciation concurrentielle (Heal 2005). Fort de ce constat, cet article se propose d'étudier cette dimension stratégique à un niveau sectoriel.

S'appuyant sur un modèle en deux étapes (Arjaliès et Ponssard 2010), qui distingue une approche dite «de conformité» (i.e. figures imposées), d'une deuxième étape qualifiée «d'opportunité » (i.e. figures libres), l'article analyse et compare deux secteurs d'activités particulièrement émetteurs en matière de $\mathrm{CO}_{2}$ : l'industrie chimique et l'industrie cimentière. L'article démontre que la capacité des entreprises à innover face au $\mathrm{CO}_{2}$ est contrainte ou facilitée par des facteurs structurels inhérents au secteur d'activité. En particulier, il identifie trois éléments clefs qui participent à expliquer pourquoi certains secteurs parviennent à construire des stratégies proactives de lutte contre le réchauffement climatique (ex : chimie) tandis que d'autres adoptent des démarches plus réactives (ex : ciment) :1) la dépendance du processus de production vis à vis des ressources naturelles, 2) la capacité à jouer sur le portefeuille d'activités et le rôle induit pour la $R \& D$ et 3) la structure du secteur aval.

L'article est structuré comme suit. Après avoir resitué notre démarche dans la littérature dans la section 2, la section 3 présente les deux secteurs d'activités retenus et les hypothèses formulées. L'analyse empirique est menée dans la section 4. A partir d'une discussion des principaux résultats, la section 5 conclut sur les implications de l'étude sur les liens entre développement durable et innovation stratégique.

\section{Les liens entre RSE et stratégie : ancrage théorique}

Trois types d'arguments ont été traditionnellement avancés dans la littérature pour justifier les choix stratégiques d'une entreprise en matière environnementale, et plus largement en termes de 
RSE. Le courant «business ethics» considère qu'une entreprise se doit d'intégrer ces problématiques en raison de son obligation morale envers la société et non pour des raisons économiques (Goodpaster 1983). Le courant «business and society (Wood 1991) affirme que l'entreprise est une institution sociale créée par la société, envers laquelle elle est redevable et qui peut mettre fin à ses activités si elles sont inappropriées. Cette approche que l'on qualifie aujourd'hui couramment de théorie de la RSE s'est fortement appuyée sur la théorie néoinstitutionnelle (DiMaggio et Powell 1983) et la théorie des parties prenantes (Freeman 1984). Selon ces auteurs, une entreprise intègre la RSE dans sa stratégie principalement en raison des contraintes institutionnelles qui pèsent sur cette dernière et qui l'obligent à répondre aux attentes de ses parties prenantes afin de préserver sa légitimité (i.e. licence to operate). Enfin, l'approche «business case» (Vogel 2005) voit dans la RSE une source d'innovation stratégique permettant d'améliorer la performance économique de l'entreprise.

Loin de s'opposer, ces trois courants se complètent. Il semble en effet raisonnable de penser que les choix stratégiques d'une entreprise en termes de RSE résultent de considérations à la fois éthiques, institutionnelles et économiques. Néanmoins, étant donné la prééminence des arguments économiques en ce qui concerne la lutte contre le réchauffement climatique (Stern 2006), nous avons choisi de nous intéresser aux arguments proposés par l'approche «business case ». Plus particulièrement, nous souhaitons mieux comprendre pourquoi certaines entreprises perçoivent dans la réduction des émissions $\mathrm{CO}_{2}$ une source d'opportunité stratégique, tandis que d'autres semblent y voir essentiellement une contrainte à gérer. Afin de pouvoir identifier ce qui pourrait justifier de telles différences, nous nous intéressons aux différents arguments qui ont été développés dans l'approche «business case ». Notre hypothèse est que les choix stratégiques en matière de réduction des émissions $\mathrm{CO}_{2}$ sont en grande partie déterminés par des caractéristiques sectorielles, dimension souvent négligée dans la littérature. 
Le courant «business case» a récemment bénéficié d'un regain d'intérêt auprès de la communauté académique et professionnelle. Ceci s'est notamment traduit par la publication de plusieurs articles dans la Harvard Business Review. Porter et Kramer (2006, 2011), par exemple, distinguent la « Responsive CSR », qui consiste à répondre aux attentes des parties prenantes et à adoucir les effets néfastes des contraintes réglementaires sur la chaine de valeur (i.e. approche risque), et la «Strategic CSR» qui vise à aller au-delà des bonnes pratiques en modifiant profondément la position stratégique de l'entreprise par rapport à ses concurrents (i.e. approche opportunité). Selon ces derniers, il existe trois façons pour une entreprise de créer ce qu'ils appellent de la «valeur partagée » (i.e. conciliant à la fois progrès économiques et sociétaux) : repenser les produits et les marchés, redéfinir les leviers de productivité le long de la chaine de valeur et permettre le développement de nouveaux réseaux économiques locaux. Dans la même veine, Nidumolu et al. (2009) encouragent les entreprises à faire de la conformité environnementale une source d'opportunité stratégique en reconfigurant l'ensemble de leur chaine de valeur et en concevant de nouveaux produits et services. Aggeri et al. (2005), quant à eux, différencient les «figures imposées» des «figures libres» du développement durable. L'espace des «figures imposées» définit les standards auxquels l'entreprise doit se conformer pour réduire les risques associés aux enjeux du développement durable. Cette approche réactive ne confère pas à l'entreprise un avantage concurrentiel. A l'inverse, les « figures libres » renvoient à un ensemble de démarches et de pratiques innovantes qui viennent interroger le cœur de métier et les orientations stratégiques de l'entreprise. Ce sont ces « figures libres » qui peuvent générer de la création de valeur en permettant le développement de nouveaux modèles économiques associés au développement durable.

Dans un article précédent, Arjaliès et Ponssard (2010) proposent une typologie de stratégies qui s'inscrit bien dans ce courant «business case». Dans ce modèle, deux types de stratégies et les conditions de passage d'un type à l'autre (cf. Tableau 1) sont distingués. Dans la première 
étape dite de «conformité » (i.e. «figures imposées »), le changement climatique apparaît comme une problématique de gestion des risques au niveau du groupe. L'entreprise cherche à limiter les changements induits par les réglementations liées au $\mathrm{CO}_{2}$, notamment au travers d'actions de lobbying, et ne perçoit pas dans la régulation une opportunité stratégique. Les systèmes de pilotage sont peu impactés par les contraintes de réduction des émissions $\mathrm{CO}_{2}$, à l'exception d'actions incrémentales, telle que l'amélioration de processus existants destinés à encourager une meilleure efficacité énergétique dans la production. Au cours de la seconde étape qualifiée d'« opportunité » (i.e. «figures libres »), l'entreprise perçoit dans la réduction des émissions $\mathrm{CO}_{2}$ une occasion de reformuler sa vision et sa culture autour d'une nouvelle stratégie. Une analyse sectorielle est conduite par le groupe afin d'identifier les forces et les faiblesses de l'entreprise par rapport à ses concurrents, clients et fournisseurs. La nouvelle orientation stratégique vise à repenser le modèle économique de l'entreprise en fonction du défi du changement climatique. Des objectifs synthétiques sont explicités et communiqués dans le rapport de Développement Durable et déclinés en plans d'action internes. Ces plans d'action sont alors explicitement introduits dans les systèmes de pilotage avec des objectifs opérationnels et des incitations correspondantes. Cette étape deux est très proche du concept de «figure libre » développé par Aggeri et al. (2005), tant au niveau de sa vision stratégique que de son positionnement concurrentiel. Elle s'en distingue néanmoins par son caractère plus micro, puisqu'elle s'intéresse également à la structure organisationnelle et aux outils de pilotage mis en place par l'entreprise afin d'atteindre ses nouveaux objectifs stratégiques.

Le passage de l'étape une basée sur la gestion des risques et la conformité à l'étape deux dont l'objectif est de générer de nouvelles innovations et opportunités stratégiques requiert notamment: 1) la reconnaissance d'une opportunité stratégique au niveau de la direction générale de l'entreprise, 2) une approche transversale R\&D, Production et Ventes pour formuler

\footnotetext{
${ }^{5}$ Conformité doit être ici entendue dans un sens plus large que le strict respect de normes et de standards.
} 
des objectifs et 3) une transformation cohérente des systèmes de pilotage faisant une place plus importante aux systèmes interactifs (i.e. favorisant l'apprentissage organisationnel, voir par exemple Simons (1995)).

\begin{tabular}{|c|c|c|}
\hline Dimension de la stratégie & $\begin{array}{l}\text { Stratégie 1: } \\
\text { Conformité }\end{array}$ & $\begin{array}{l}\text { Stratégie 2: } \\
\text { Opportunité }\end{array}$ \\
\hline $\begin{array}{l}\text { A. Vision } \\
\text { Système de Valeur }\end{array}$ & 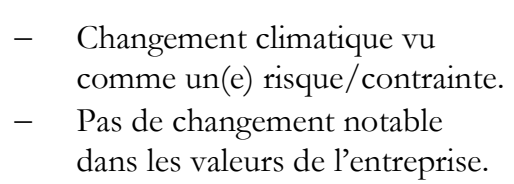 & $\begin{array}{l}\text { - Changement climatique vu } \\
\text { comme une opportunité. } \\
\text { - Changement global dans les } \\
\text { valeurs de l'entreprise. }\end{array}$ \\
\hline $\begin{array}{l}\text { B. Nature des engagements } \\
\text { industriels et de marché en } \\
\text { termes de } \mathrm{CO}_{2}\end{array}$ & $\begin{array}{ll}- & \text { Emissions en intensité. } \\
- & \text { Pas d'engagements sur les } \\
\text { nouveaux produits ou services } \\
\text { (seulement des projets en } \\
\text { cours). }\end{array}$ & $\begin{array}{l}\text { - } \quad \text { Emissions en valeurs absolues. } \\
\text { - Nouveaux produits et services } \\
\text { avec des partenariats, incluant } \\
\text { des ONG. }\end{array}$ \\
\hline $\begin{array}{l}\text { C. Position vis-à-vis de la } \\
\text { réglementation sur les émissions } \\
\text { de } \mathrm{CO}_{2}\end{array}$ & $\begin{array}{ll}\text { - } & \text { Promotion des accords } \\
\text { sectoriels. } \\
\text { - } & \text { A défaut limiter l'impact des } \\
\text { systèmes cap and trade } \\
\text { unilatéraux (allocations } \\
\text { gratuites). }\end{array}$ & $\begin{array}{l}\text { - Promotion des systèmes cap } \\
\text { and trade. } \\
\text { - } \quad \text { Prix unique du carbone au } \\
\text { niveau mondial. }\end{array}$ \\
\hline D. Portefeuilles d'activités & $\begin{array}{l}\text { - Pas de changement notable } \\
\text { dans le portefeuille d'activités. }\end{array}$ & $\begin{array}{ll}\text { - } & \text { Repositionnement du } \\
& \text { portefeuille d'activités. } \\
\text { - } & \text { Enjeu sur l'ensemble de la } \\
\text { chaine de valeur. }\end{array}$ \\
\hline $\begin{array}{l}\text { E. Le processus de pilotage de } \\
\text { ces engagements }\end{array}$ & $\begin{array}{ll}\text { - } & \text { Renforcement des leviers } \\
\text { existants (ex: efficacité } \\
\text { énergétique). }\end{array}$ & $\begin{array}{ll}\text { - } & \text { Nouvelle organisation avec } \\
\text { implication dans les revues } \\
\text { stratégiques des business units. } \\
\text { - } & \text { Centres de partage d'expérience } \\
& \text { (aspect transversal de } \\
& \text { l'innovation produit). } \\
- & \text { Impacts sur les rémunérations. }\end{array}$ \\
\hline
\end{tabular}

\section{Tableau 1 - Proposition d'un modèle stratégique (d'après Arjaliès et Ponssard, 2010)}

Alors que la dimension sectorielle est habituelle dans les analyses stratégiques concurrentielles menées dans les entreprises depuis le textbook de Porter (1980), on s'aperçoit qu'elle reste largement inexplorée dans les différentes approches stratégiques de la RSE, qui préfèrent mettre l'accent sur le potentiel de transformation de l'organisation (Aggeri et al. 2005). Ceci est d'autant

\footnotetext{
${ }^{6}$ Marchés d'échange de permis négociables.
} 
plus étonnant que l'analyse extra-financière effectuée par les sociétés de gestion d'actifs au service d'une meilleure performance financière s'appuie quasiment toujours sur une approche sectorielle. Fort de ce constat, ce papier cherche à mieux comprendre quels sont les impacts potentiels du secteur sur les différentes dimensions de la stratégie identifiées dans le modèle précédent (cf. Tableau 1). En raison des contraintes méthodologiques, nous avons souhaité nous consacrer aux cinq premières dimensions (A à D), laissant l'analyse des processus de pilotage à des études ultérieures.

L'analyse stratégique sectorielle que nous proposons s'appuie classiquement sur l'identification des principales étapes de valeur ajoutée, l'importance et la nature du $\mathrm{CO}_{2}$ à chacune de ces étapes, et les leviers possibles en termes de réduction du $\mathrm{CO}_{2}$. Nous considérons aussi une approche plus spécifique qui combine les caractéristiques économiques sectorielles avec des enjeux sociétaux (Hommel et Godard 2001). Ces auteurs se sont intéressés à la question suivante: pourquoi les comportements proactifs en matière de RSE sont-ils loin d'être homogènes ? Leur hypothèse est que le niveau de pro-activité ne peut pas simplement s'expliquer par la perte potentielle de légitimité (licence to operate) suite à une contestation de la société civile. C’est l'existence d'investissements lourds non récupérables (au sens de la théorie des marchés contestables de Baumol et al. (1982)), par exemple la construction d'une usine à longue durée de vie et non dé-localisable, qui conduit à s'engager dans des actions majeures en matière de RSE.

\section{Influence du secteur d'activité sur les choix stratégiques en matière de $\mathbf{C O}_{2}$}

\subsection{Le rôle du $\mathrm{CO}_{2}$ dans une analyse sectorielle : risque versus opportunité}

Une analyse stratégique démarre habituellement en positionnant le secteur étudié vis à vis de cinq «forces concurrentielles »: amont, aval, entrants, substituts, et concurrence interne (Porter 
1980). Nous appliquons cette grille pour identifier l'impact du $\mathrm{CO}_{2}$ sur les marges de manœuvre et les contraintes qui pèsent sur l'ensemble des entreprises dans les deux secteurs étudiés. Notre analyse s'appuie sur les figures 1 et 2 .

Le secteur cimentier est relativement simple à étudier. Le produit est homogène, peu coûteux à produire par rapport à son coût de transport terrestre. Les coûts de transport par voie maritime sous forme de vrac sont peu élevés et les équilibres régionaux peuvent être largement affectés par les flux correspondants. Les grandes entreprises du secteur sont des multinationales présentes dans de nombreux pays, qui tirent parti de leur réseau d'usines pour optimiser leur production. Le processus de production en tant que tel est bien identifié - la production de clinker - qui constitue également la principale source de $\mathrm{CO}_{2}$ (de par la décarbonatation du calcaire, et de par l'énergie nécessaire à cette opération à haute température). Le ciment proprement dit s'obtient par broyage et ajout de différents composants au clinker : notamment laitier et cendres volantes. Au niveau final, le ciment constitue une part importante des composants dans trois marchés : infrastructures, bâtiments industriels et commerciaux, maisons individuelles. Pour ces deux derniers segments de marché, l'enjeu du $\mathrm{CO}_{2}$ tient non seulement à l'empreinte carbone des composants mais aussi à l'efficacité énergétique tout au long de leur cycle de vie (y compris l'empreinte carbone associée aux inputs en amont de l'industrie cimentière). Selon les statistiques de l'AIE (2004), en termes de scope 1, le secteur cimentier représentait en 2000 environ 18\% des émissions mondiales industrielles de $\mathrm{CO}_{2}$ (Baumert et al. 2005). ${ }^{7}$

Les principaux leviers pour limiter les émissions portent sur le processus de production de clinker (meilleure efficacité énergétique, modification du mix de combustibles), l'augmentation

\footnotetext{
${ }^{7}$ Le scope 1 comprend l'ensemble des émissions directes de GES, le scope 2 inclut les émissions indirectes liées à la consommation d'énergie, le scope 3 incorporant toutes les autres émissions indirectes.
} 
des ajouts au clinker, les changements éventuels de produits et de procédés pour améliorer l'efficacité énergétique au niveau de la construction proprement dite.

Les possibilités de se différencier en termes de $\mathrm{CO}_{2}$ au niveau de la production de ciment apparaissent faibles : forte homogénéité du processus de production du clinker, technologie mûre, disponibilité limitée des sources d'ajouts. A priori les sources de différenciation semblent plus importantes en aval. Par contre, ce niveau est très fragmenté : nombreux intervenants correspondants à différents corps de métier, poids des facteurs régionaux, multiplicité des réglementations.

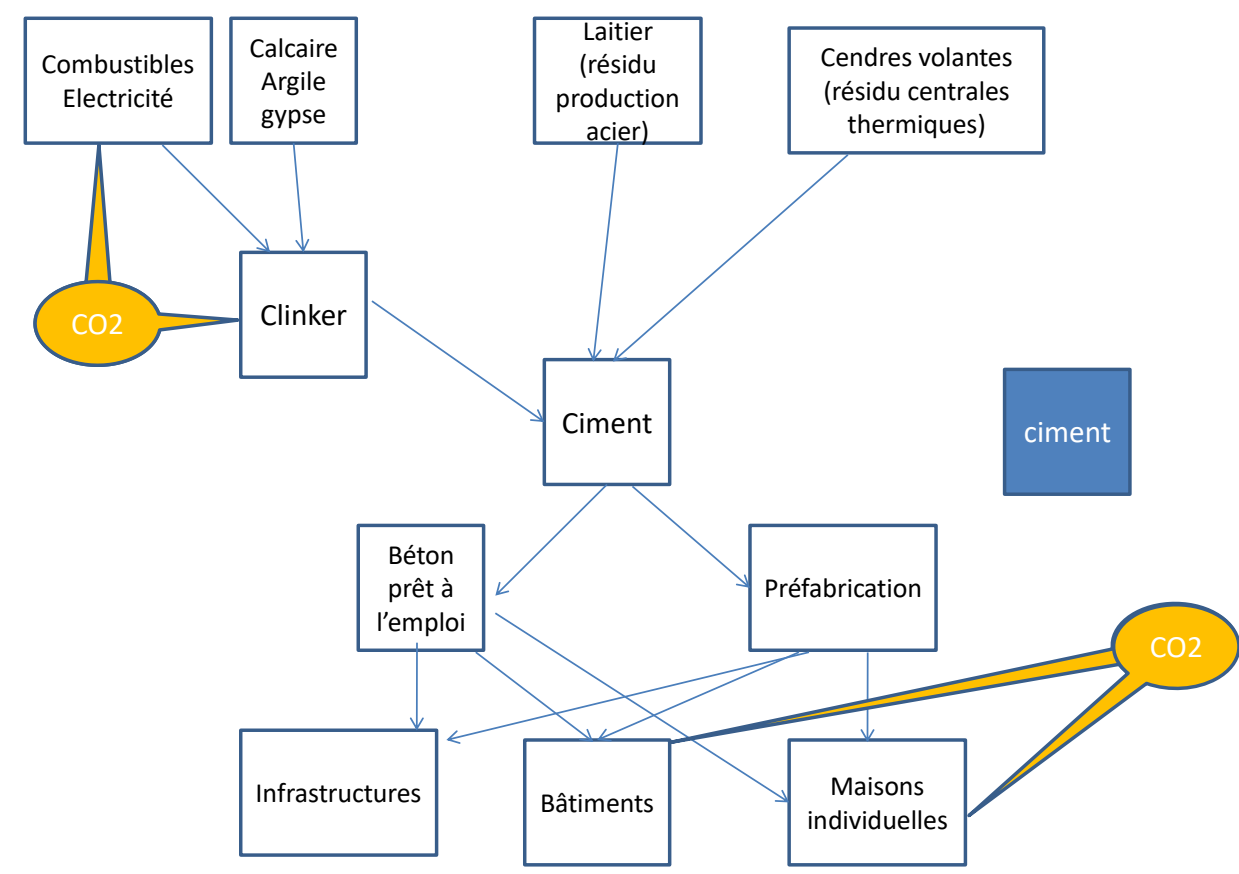

Figure 1 - Chaine de valeur du secteur cimentier

Le secteur chimique est beaucoup plus complexe. Il comprend de nombreux processus de production allant de la chimie de base (ammoniaque, benzène, éthylène, propylène...) à des processus différenciés par type d'applications : chimie de spécialités, chimie dite life science, chimie 
de grande consommation. D’après les statistiques du CEFIC (European Chemical Industry Council), la chimie de base représentait 60\% des ventes du secteur européen en 2009. Les entreprises sont très hétérogènes en termes de positionnement sur ces différents métiers et en taille.

Il faut noter que la plupart des grandes entreprises du secteur présentent leurs activités par marchés. A ce titre, on trouve à côté des segments présentés ici des secteurs d'applications comme l'automobile, l'agriculture, l'électronique ou l'ensachage... Les enjeux propres à ces marchés impliquent un renouvellement rapide des produits, si bien que la $R \& D$ tient un rôle majeur dans le secteur. Les dépenses de R\&D représentent 4\% des ventes (source : CEFIC), alors que ce pourcentage est inférieure à $1 \%$ pour l'industrie cimentière (sources rapports annuels).

L'enjeu du $\mathrm{CO}_{2}$ et les leviers correspondants tiennent aux inputs (matières fossiles qui peuvent en partie être substituées par des produits issus de la biomasse faible en carbone, et ceci dans des proportions bien plus fortes que pour l'industrie cimentière) et à l'énergie nécessaire aux différents stades de production (efficacité énergétique). En termes de scope 1, toujours selon les statistiques de l'AIE, le secteur chimie et pétrochimie représentait en 2000 environ 23\% des émissions mondiales industrielles de $\mathrm{CO}_{2}$. De par son positionnement amont, l'industrie chimique est aussi en mesure de jouer sur l'empreinte carbone de ses clients. Lorsque ces clients sont de grosses entreprises, elle est en mesure de développer des applications susceptibles de toucher rapidement des marchés importants pour lesquels les produits et solutions s'appuient souvent sur des joint ventures avec des partenaires puissants. 


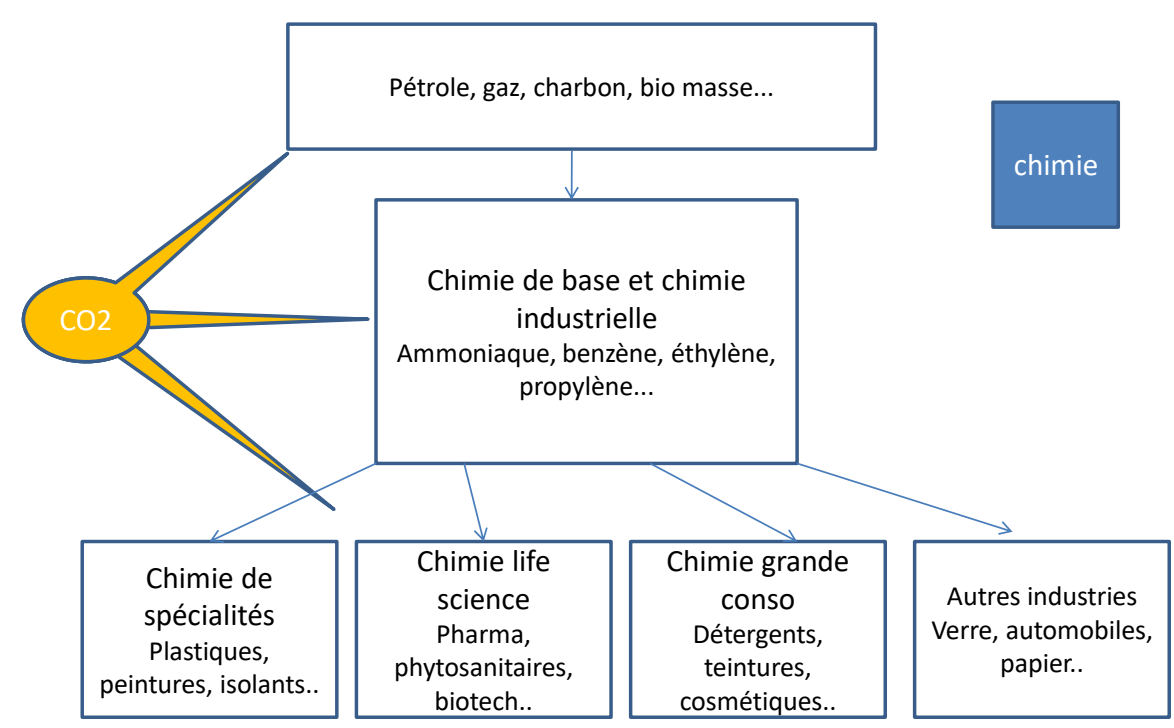

Figure 2 - Chaine de valeur du secteur chimique

Cette analyse sectorielle nous amène à formuler une première hypothèse.

Hypothèse 1: L'enjeu du $\mathrm{CO}_{2}$ est principalement centré sur le processus de production pour l'industrie cimentière. Il est plus diffus pour l'industrie chimique : processus de production mais aussi impacts possibles sur l'ensemble du portefeuille d'activités de l'entreprise aussi bien en amont qu'en aval.

\subsection{Le rôle du $\mathrm{CO}_{2}$ dans une analyse sectorielle : contestabilité économique et sociale}

Nous reprenons ici la démarche développée par Godard et Hommel (2001). En ce qui concerne les enjeux liés de contestabilité économique et sociale liés au $\mathrm{CO}_{2,}$, on pourrait a priori penser qu’il n'existe pas de différence notable entre le secteur chimique et cimentier. En effet, les entreprises de ces secteurs devraient a priori s'engager dans des stratégies RSE proactives de manière à protéger le caractère fortement irréversible de leurs investissements industriels, hautement capitalistiques. 
Or, dans les faits, il existe entre ces deux secteurs des différences importantes, qui résultent principalement de la place qu'occupe le $\mathrm{CO}_{2}$ dans l'ensemble des effets externes générés par ces deux activités. D'une part, le ciment émet surtout du $\mathrm{CO}_{2}$ et peu d'autres gaz à effet de serre tandis que l'industrie chimique émet toute une série de GES qui en équivalent $\mathrm{CO}_{2}$ ont des facteurs multiplicatifs allant au-delà de 10 000, par exemple pour le HFC, mais qui surtout peuvent avoir des effets extrêmement toxiques comme en témoignent des catastrophes restées dans la mémoire collective. D'autre part, les effets négatifs directement visibles associés à la production de ciment concernent surtout l'exploitation de carrières gigantesques générant toute une série de nuisances pendant leur exploitation (circulation d'engins, dynamitage des roches, etc...) et susceptibles de laisser des trous béants dans le paysage après l'exploitation. La politique proactive dans ce secteur s'est donc plutôt tournée vers la maitrise de ces enjeux de contestation sociale.

Ces considérations nous amènent à formuler notre deuxième hypothèse.

Hypothèse 2: Les enjeux du $\mathrm{CO}_{2}$ vont naturellement s'intégrer dans une politique proactive large dans le secteur de la chimie tandis qu'ils resteront circonscrits pour l'industrie cimentière.

\section{Méthodologie}

Cet article est basé sur des études de cas exploratoires visant à comparer dans chaque secteur d'activité 6 à 7 multinationales (cf. tableau 2). Non statistiquement représentatives, les entreprises furent sélectionnées en fonction de leur rôle dans leur secteur (i.e. rôle de leader), de leur pays d’origine (i.e. diversité géographique). L’analyse s'est effectuée en trois étapes: 
- Nous avons d'abord identifié les principaux enjeux en conduisant une étude exploratoire auprès de DuPont et Lafarge. Cette étude s'est appuyée sur 1) des entretiens et des réunions avec plusieurs dirigeants en charge de la stratégie et/ou des questions liées au $\left.\mathrm{CO}_{2}, 2\right)$ des documents internes mis à notre disposition et 3) des sources secondaires telles que des documents institutionnels, le CDP (Carbon Disclosure Project ${ }^{8}$ ) et des analyses d'agences de notation sociétale. Nous avons construit notre modèle théorique (cf. Tableau 1) à partir de ces informations.

- Nous avons ensuite effectué une analyse comparative des stratégies $\mathrm{CO}_{2}$ sur un horizon temporel de 20 ans nous permettant d'observer de réelles évolutions dans la conduite stratégique des groupes étudiés. La limitation des données disponibles nous a menéà établir notre cadre d'étude sur une période allant de 2000 à 2009 pour les données des rapports annuels et à partir de 1990 - ou année de référence - pour les émissions $\mathrm{CO}_{2}$. En vue de valider nos hypothèses, le traitement des données fut effectué selon la méthodologie suivante :

1. Nous avons récolté les données financières des entreprises étudiées au sein des rapports annuels d'activité disponibles sur la période 2000 -2009. A partir de ces données, nous avons étudié tant l'évolution du chiffre d'affaire global que sa structure même - cœurs de métier, zones géographiques. Les investissements en $\mathrm{R} \& \mathrm{D}$ ainsi que les émissions annuelles de $\mathrm{CO}_{2}$ nettes ou en intensité par biens produits ont aussi été relevées.

2. La $2^{\text {ème }}$ étape a consisté en l'observation de ces données économiques en les associant aux émissions de $\mathrm{CO}_{2}$ déclarées - global et / ou en intensité par

\footnotetext{
${ }^{8}$ Le Carbon Disclosure Project est une ONG au service d'investisseurs institutionnels qui collecte auprès de 2500 multinationales des informations sur leurs visions, stratégies, plans d'actions et résultats vis-à-vis des émissions $\mathrm{CO}_{2}$. L'ensemble des données recueillies par le $\mathrm{CDP}$ sont publiques.
} 
produit - sur la même période au regard des engagements de réductions des émissions de $\mathrm{CO}_{2}$ pris par ces entreprises.

3. La $3^{\text {ème }}$ étape a porté sur la validation de la correspondance des informations trouvées en étape 1 et 2 avec les réponses données par le CDP.

4. Enfin, dernière étape, nous avons analysé l'évolution des discours des dirigeants grâce à l'utilisation de mots clefs afin de voir l'évolution de la vision de l'entreprise depuis les premiers rapports de développement durable disponibles jusqu'aux plus récents - 2009 .

\begin{tabular}{|l|c|l|c|}
\hline \multicolumn{1}{|c|}{ Industrie cimentière } & $\begin{array}{c}\text { Emissions nettes } \\
\text { totales en 2009(Mt) }\end{array}$ & \multicolumn{1}{|c|}{ Industrie chimique } & $\begin{array}{r}\text { Emissions nettes } \\
\text { totales en 2009 (Mt) }\end{array}$ \\
\hline Lafarge & 92,0 & DuPont & 16,2 \\
Holcim & 89.3 & Bayer r & 8,1 \\
Cemex & 39,7 & Bayer Material Science & 4,8 \\
Italcementi & 36,3 & Bayer Health Care & 0,5 \\
Heildeberg & 52,8 & Bayer Crop Science & 1,1 \\
CRH & 9,8 & Rhodia & 5,3 \\
Titan & 8,7 & BASF & 29,3 \\
& & AkzoNobel & 4,6 \\
& & Dow Chemical & 41.3 \\
\hline
\end{tabular}

Tableau 2 - Entreprises analysées dans chaque secteur d'activité

(Sources: Rapports annuels d'activités 2009, rapports DD 2009, sites internet et CDP)

\section{Analyse empirique}

La démarche qui suit consiste à reprendre les quatre dimensions de la stratégie telles qu'identifiées dans le tableau 1, et à tester la plus ou moins grande proximité des entreprises sur ces dimensions. Schématiquement, nous mettrons en évidence que les entreprises du secteur cimentier semblent plutôt suivre une figure «imposée » caractéristique de l'étape une, tandis que les entreprises de l'industrie chimique paraissent plus « libres » de s'engager dans des stratégies d'innovation. Nous 
reviendrons dans la section suivante sur l'apport de ces résultats pour valider les hypothèses 1 et 2.

\subsection{Vision et système de valeur (dimension $\mathrm{A})$}

Pour appréhender la vision de chaque entreprise, nous procédons à une analyse des Lettres des Présidents en introduction aux rapports Développement Durable de 2000 à 2009. Nous effectuons d'abord une analyse quantitative basée sur la présence de certains mots clés sur trois types d'enjeux :

- Place du changement climatique et des enjeux associés au $\mathrm{CO}_{2}$.

- Place de l'innovation.

- Place des enjeux liés au marché, et plus généralement à la chaine de valeur.

Les résultats correspondants sont reproduits dans les tableaux 3a et 3b. Par ailleurs, une analyse plus qualitative (notre perception des messages forts figurant dans ces lettres) nous permet de donner un sens plus synthétique à cette vision chiffrée.

Notre analyse tend à montrer qu'au niveau du secteur cimentier, ce sont plus particulièrement Holcim et CRH qui mettent en avant le changement climatique comme un enjeu sociétal global en insistant sur l'importance de la construction durable pour maitriser les émissions $\mathrm{CO}_{2}$ en améliorant la performance énergétique des bâtiments. Lafarge est aussi présent sur ce thème mais cela ne se reflète pas directement dans sa vision qui reste marquée par les valeurs traditionnelles du groupe. Au niveau du secteur chimique, BASF et DuPont associent le $\mathrm{CO}_{2}$ à des enjeux sociétaux globaux. C'est aussi le cas pour Bayer même si cela ne ressort pas de l'analyse quantitative. L'investissement du groupe Bayer dans des initiatives de meilleure gestion de la production agricole en Inde ou le programme Ecobuilding en partenariat avec des acteurs de la 
construction en sont quelques exemples. Ces programmes répondent aux problématiques globales de l'alimentation face au changement climatique et à l'urbanisation grandissante dans les pays en voie de développement.

D’une manière générale, nous constatons que les préoccupations du secteur chimique concernent presqu'exclusivement des enjeux de marchés (soit 8,57 pour les enjeux de marché versus 1,38 pour les enjeux généraux liés au changement climatique) tandis que le secteur cimentier fait état de préoccupations également réparties (soit 2.55 versus 2.28). L'innovation en tant que telle apparait beaucoup plus nettement comme un enjeu pour le secteur chimique que pour le secteur cimentier (1,08 versus 0,37$)$.

\begin{tabular}{|c|c|c|c|c|c|c|c|}
\hline & Holcim & Lafarge & Cemex & Italcementi & Heidelberg & CRH & Moyenne \\
\hline $\begin{array}{l}\text { Changement } \\
\text { climatique / } \\
\text { Protection }\end{array}$ & $1.00^{9}$ & 1.00 & 0.50 & 0.50 & 1.17 & 3.39 & 1.26 \\
\hline $\mathrm{CO}_{2}$ ou GES & 3.25 & 0.50 & 0.25 & - & 0.33 & 1.78 & 1.02 \\
\hline Total $\mathrm{CO}_{2}$ & 4.25 & 1.5 & 0.75 & 0.50 & 1.50 & 5.17 & 2.28 \\
\hline Innovation & 0.25 & 0.50 & - & 1.00 & 0.33 & 0.11 & 0.37 \\
\hline $\begin{array}{c}\text { Nouveaux } \\
\text { produits / } \\
\text { Opportunités }\end{array}$ & 0.50 & 0.50 & 0.75 & 0.75 & 0.92 & 1.31 & 0.79 \\
\hline $\begin{array}{c}\text { Consommateurs / } \\
\text { Clients }\end{array}$ & 1.00 & 0.25 & 0.50 & 0.50 & 0.17 & 1.39 & 0.63 \\
\hline Fournisseurs & 0.25 & - & 0.25 & 0.50 & 0.17 & 1.39 & 0.43 \\
\hline Marché & 0.25 & 0.50 & 0.25 & 1.00 & 0.67 & 1.56 & 0.70 \\
\hline Total marché & 2.00 & 1.25 & 1.75 & 2.75 & 1.92 & 5.64 & 2.55 \\
\hline
\end{tabular}

Tableau 3a - Analyse des Lettres de Présidents des entreprises cimentières

\begin{tabular}{|c|c|c|c|c|c|c|}
\cline { 2 - 7 } \multicolumn{1}{c|}{} & BASF & Bayer & DuPont & Akzo & Rhodia & Moyenne \\
\hline $\begin{array}{c}\text { Changement } \\
\text { climatique / } \\
\text { Protection }\end{array}$ & 0.75 & 3.00 & 1.00 & - & 0.25 & 1.00 \\
\hline CO $_{2}$ ou GES & - & 0.33 & - & 0.33 & 1.25 & 0.38 \\
\hline Total CO & $\mathbf{0 . 7 5}$ & $\mathbf{3 . 3 3}$ & $\mathbf{1 . 0 0}$ & $\mathbf{0 . 3 3}$ & $\mathbf{1 . 5 0}$ & $\mathbf{1 . 3 8}$ \\
\hline Innovation & $\mathbf{2 . 5 0}$ & $\mathbf{0 . 6 7}$ & $\mathbf{0 . 5 0}$ & $\mathbf{1 . 0 0}$ & $\mathbf{0 . 7 5}$ & $\mathbf{1 . 0 8}$ \\
\hline Nouveaux produits & 1.75 & 1.67 & 3.00 & 3.67 & 0.75 & 2.17 \\
\hline
\end{tabular}

${ }^{9}$ Pour Holcim, par exemple, le terme « changement climatique » ou « Protection du climat » est cité en moyenne une fois dans chacun des documents de l'entreprise. 


\begin{tabular}{|c|c|c|c|c|c|c|}
\hline / Opportunités & & & & & & \\
\hline $\begin{array}{c}\text { Consommateurs / } \\
\text { Clients }\end{array}$ & 4.75 & 0.33 & 2.50 & 3.00 & 2.50 & 2.62 \\
\hline Fournisseurs & 0.75 & 1.00 & - & - & - & 0.35 \\
\hline Marché & 3.50 & - & 5.00 & 6.67 & 2.00 & 3.43 \\
\hline Total market & $\mathbf{1 0 . 7 5}$ & $\mathbf{3 . 0 0}$ & $\mathbf{1 0 . 5 0}$ & $\mathbf{1 3 . 3 3}$ & $\mathbf{5 . 2 5}$ & $\mathbf{8 . 5 7}$ \\
\hline
\end{tabular}

\section{Tableau 3b - Analyse des Lettres de Présidents des entreprises chimiques}

(Sources: lettres des présidents Rapports Annuels ou Développement Durable 2000 - 2009)

\subsection{Nature des engagements en termes de $\mathrm{CO}_{2}($ dimension $\mathrm{B})$}

Nous identifions maintenant les objectifs quantifiés qui sont mentionnés dans les rapports $\mathrm{DD}$, en ce qui concerne directement les enjeux liés au changement climatique et au $\mathrm{CO}_{2}$. Nous recherchons la présence de deux types d'objectifs :

- Des objectifs industriels liés directement aux émissions ;

- Des objectifs liés à des produits ou solutions destinés à l'ensemble de la chaine de valeur.

Les tableaux $4 \mathrm{a}$ et $4 \mathrm{~b}$ reprennent le premier type d'objectifs. Chaque entreprise formule ses objectifs dans un cadre pluriannuel : une cible est annoncée à une certaine date (la date d'engagement), pour une date future, en référence à un niveau atteint dans le passé (pas forcément la date à laquelle est pris l'engagement, par exemple l'année 1990 est souvent retenue en référence aux engagements de Kyoto), puis la nature de l'engagement est précisée (en intensité ou en valeur absolue) avec l'objectif quantitatif correspondant. Chaque année, l'entreprise communique sa progression par rapport à la cible. 
En ce qui concerne le secteur cimentier, les objectifs se caractérisent par un engagement en intensité $^{10}$ et un faible écart quant au niveau annualisé de l'objectif cible (calculé comme le pourcentage de réduction divisé par le nombre d'années entre la référence et la cible). Les réductions effectives des émissions à l'année 2009 sont assez homogènes, à l'exception notable d'Italcementi.

Pour le secteur chimique, nous notons tout d'abord que les engagements figurant dans les rapports 2009 sont issus d'engagements plus récents que pour l'industrie cimentière. Ceci amène à penser qu'il s'agit en fait du renouvellement d'engagements plus anciens. On note aussi une plus forte hétérogénéité : la nature des objectifs se partage entre objectifs en valeur absolue et en intensité. Les objectifs annualisés correspondants portent sur des fourchettes plus larges que pour l'industrie cimentière et les résultats 2009 sont nettement plus dispersés. On note que certaines entreprises du secteur attribuent à la crise financière de 2009 leurs mauvais résultats en intensité (en arguant des émissions fixes liées à leurs opérations en dépit de volumes plus faibles). On peut aussi noter la grande difficulté des entreprises à anticiper leurs résultats en valeur absolue. De tels engagements apparaissent donc comme des engagements politiques destinés à donner un signal fort quant à la volonté de l'entreprise de déconnecter sa croissance du niveau de ses émissions.

Suite à notre étude exploratoire (cf. section 3), nous nous attendions à trouver des engagements en termes de produits/marchés chez les chimistes. Nous avions noté que dès 2005, DuPont s'était engagé (à l'horizon 2015) à (i) un doublement de ses investissements R\&D sur des projets en relation directe avec un gain environnemental pour ses clients, (ii) à augmenter de 2 MM de dollars ses revenus sur des produits faisant baisser l'empreinte carbone ou l'efficacité énergétique chez ses clients; (iii) à doubler ses revenus sur des produits issus de ressources

${ }^{10}$ A l'exception de Lafarge qui du fait de son partenariat avec WWF a aussi un objectif en valeur absolue pour ses émissions dans les pays industrialisés, mais il ne semble pas que cet engagement ait joué un rôle important sur le plan opérationnel car très contingent à l'évolution du marché européen (source : entretien Lafarge). 
renouvelable (chimie verte). Ces objectifs sont repris et déclinés en sous-objectifs dans le rapport de DuPont en 2010 (pas de rapport DD en 2009).

Nous n'avons pas retrouvé cette même visibilité quant à la vision «marché » chez les autres chimistes. Seule Akzo Nobel formule des objectifs marchés (30\% des ventes à partir de produits « eco-premium »). Les autres entreprises communiquent bien autour de certains produits verts, ou bien font état de partenariat pour mettre au point des solutions nouvelles, par exemple autour du projet Eco-Efficiency Building (WBCSD), mais prennent peu d'engagements précis en ce domaine. Les entreprises du secteur cimentier communiquent également sur ce thème mais aucune d'entre elles ne fait état d'objectifs quantifiés en la matière. ${ }^{11}$

\begin{tabular}{|c|c|c|c|c|c|c|}
\hline \multirow{2}{*}{ Entreprise } & $\begin{array}{c}\text { Date } \\
\text { d'engagement }\end{array}$ & $\begin{array}{c}\text { Date cible } \\
\text { / Année de } \\
\text { référence }\end{array}$ & $\begin{array}{c}\text { Nature de la } \\
\text { cible }\end{array}$ & $\begin{array}{c}\text { Objectif } \\
\text { cible / } \\
\text { Référence }\end{array}$ & $\begin{array}{c}\text { Obj/An } \\
\text { Intensité }\end{array}$ & $\begin{array}{c}\text { Résultat 2009 } \\
\text { / Cible 2009 }\end{array}$ \\
\hline Lafarge & 2000 & $2010 / 1990$ & Intensité & $20 \%$ & $1 \%$ & $112 \%$ \\
\hline \multirow{2}{*}{ Holcim } & 2002 & $2010 / 1990$ & Intensité & $20 \%$ & $1 \%$ & $111 \%$ \\
\cline { 2 - 7 } & 2010 & $2015 / 1990$ & Intensité & $25 \%$ & $1 \%$ & \\
\hline \multirow{2}{*}{ Cemex } & 2005 & $2015 / 1990$ & Intensité & $25 \%$ & $1 \%$ & $115 \%$ \\
\hline Italcementi & 2002 & $2012 / 1990$ & Intensité & $2 \%$ & $0,1 \%$ & $-49 \%$ \\
\hline \multirow{2}{*}{ Heidelberg } & 2007 & $2012 / 1990$ & Intensité & $5 \%$ & $0,2 \%$ & $-20 \%$ \\
\hline CRH & 2003 & $2010 / 1990$ & Intensité & $15 \%$ & $0,8 \%$ & $137 \%$ \\
\hline \multirow{2}{*}{ Titan } & 2007 & $2015 / 1990$ & Intensité & $15 \%$ & $0,6 \%$ & $62 \%$ \\
\hline \multirow{2}{*}{ CR } & 2005 & $2010 / 1990$ & Intensité & $15 \%$ & $0,6 \%$ & $141 \%$ \\
\hline
\end{tabular}

Tableau 4a - Analyse des objectifs et résultats de réduction des émissions de $\mathrm{CO}_{2}$ des entreprises cimentières

\begin{tabular}{|c|c|l|l|l|l|l|l|l|}
\hline Entreprise & $\begin{array}{l}\text { Date } \\
\text { d'engagement }\end{array}$ & $\begin{array}{l}\text { Date } \\
\text { cible / } \\
\text { Année de } \\
\text { référence }\end{array}$ & $\begin{array}{l}\text { Nature } \\
\text { de la } \\
\text { cible }\end{array}$ & $\begin{array}{l}\text { Objectif } \\
\text { cible / } \\
\text { Référence }\end{array}$ & $\begin{array}{l}\text { Obj/An } \\
\text { Intensité }\end{array}$ & $\begin{array}{l}\text { Résultat } \\
\text { 2009/ } \\
\text { Cible } \\
\text { 2009 }\end{array}$ & $\begin{array}{l}\text { Obj/An } \\
\text { Absolue }\end{array}$ & $\begin{array}{l}\text { Résultat } \\
\text { 2009/ } \\
\text { Cible } \\
\text { 2009 }\end{array}$ \\
\hline Basf & 2008 & $2020 / 2002$ & Intensité & $25 \%$ & $1,4 \%$ & $0 \%$ & & \\
\hline $\begin{array}{c}\text { Dow } \\
\text { Chemical }\end{array}$ & 2006 & $2015 / 2005$ & Intensité & $25 \%$ & $2,5 \%$ & $0 \%$ & \\
\hline
\end{tabular}

${ }^{11}$ Noter aussi qu'il y a une certaine ambiguïté quant à la définition de produits verts dans le ciment. Les ajouts de laitier et de cendres volantes peuvent-elles être considérés comme « carbon free ». Cette question est en débat dans l'industrie (communication privée avec Cembureau). 


\begin{tabular}{|c|c|c|c|c|c|c|c|c|}
\hline Entreprise & $\begin{array}{l}\text { Date } \\
\text { d'engagement }\end{array}$ & $\begin{array}{l}\text { Date } \\
\text { cible / } \\
\text { Année de } \\
\text { référence }\end{array}$ & $\begin{array}{l}\text { Nature } \\
\text { de la } \\
\text { cible }\end{array}$ & $\begin{array}{l}\text { Objectif } \\
\text { cible / } \\
\text { Référence }\end{array}$ & $\begin{array}{l}\text { Obj/An } \\
\text { Intensité }\end{array}$ & $\begin{array}{l}\text { Résultat } \\
2009 \text { / } \\
\text { Cible } \\
2009\end{array}$ & $\begin{array}{l}\text { Obj/An } \\
\text { Absolue }\end{array}$ & $\begin{array}{l}\text { Résultat } \\
2009 / \\
\text { Cible } \\
2009\end{array}$ \\
\hline \multirow{3}{*}{$\begin{array}{c}\text { Bayer } \\
\text { material } \\
\text { science } \\
\text { - } \text { Bayer } \\
\text { Heath Care } \\
\text { Bayer Crop } \\
\text { Science }\end{array}$} & 2007 & $2020 / 2005$ & Intensité & $25 \%$ & $1,7 \%$ & $30 \%$ & & \\
\hline & 2007 & $2020 / 2005$ & $\begin{array}{l}\text { Valeur } \\
\text { absolue }\end{array}$ & $5 \%$ & & & $0,2 \%$ & $1125 \%$ \\
\hline & 2007 & $2020 / 2005$ & $\begin{array}{l}\text { Valeur } \\
\text { absolue }\end{array}$ & $15 \%$ & & & $1 \%$ & $275 \%$ \\
\hline DuPont & 2005 & $2015 / 2004$ & $\begin{array}{l}\text { Valeur } \\
\text { absolue }\end{array}$ & $15 \%$ & & & $1,4 \%$ & $147 \%$ \\
\hline \multirow[b]{2}{*}{$\begin{array}{l}\text { Akzo } \\
\text { Nobel }\end{array}$} & 2009 & $2015 / 2009$ & $\begin{array}{l}\text { Valeur } \\
\text { absolue }\end{array}$ & $10 \%$ & & & $1,7 \%$ & \\
\hline & 2009 & $2015 / 2009$ & $\begin{array}{c}\text { Intensité } \\
(20 \text { à } \\
25 \%)\end{array}$ & $25 \%$ & $4,2 \%$ & & & \\
\hline Rhodia & 2002 & $2010 / 1990$ & $\begin{array}{l}\text { Valeur } \\
\text { absolue }\end{array}$ & $30 \%$ & & & $1,5 \%$ & $274 \%$ \\
\hline
\end{tabular}

Tableau $4 \mathrm{~b}$ - Analyse des objectifs et résultats de réduction des émissions de $\mathrm{CO}_{2}$ des entreprises chimiques

(Sources : Rapport Annuels et DD 2000-2009 + sites internet)

\subsection{Position de l'entreprise vis-à-vis de la réglementation $\mathrm{CO}_{2}$ (dimension $\mathrm{C}$ )}

Toutes les entreprises cimentières étudiées sont membres du CSI (Cement Sustainability Initiative). Le CSI regroupe 23 entreprises du secteur, ce qui représente environ $1 / 3$ des émissions totales. Le CSI est l'un des programmes majeurs du WBCSD. L'objectif de ce programme qui a démarré en 2000 est notamment de développer une Technology Road Map identifiant les leviers permettant de limiter les émissions de $\mathrm{CO}_{2}$ à l'horizon 2030. En termes de régulation, le CSI cherche à peser sur les différentes politiques nationales en soulignant les risques associés à la mise en place de prix du carbone inégaux dans les différentes zones géographiques (Europe, Amérique du Nord, pays émergents, etc.). Ces risques portent, d'une part, sur les enjeux de compétitivité compte tenu de l'impact de ce prix sur le coût de production (les cimentiers craignent que le commerce international soit profondément affecté) et, d'autre part, sur les enjeux de fuites de 
carbone liées au déplacement de la production vers les zones les plus laxistes en matière de régulation.

Le CSI préconise la mise en place d'accords sectoriels associant des objectifs en intensité dans les pays émergents avec un système de cap and trade dans les pays industriels mais associé à l'octroi d'allocations gratuites pour les industriels du secteur cimentier, allocations basées sur des benchmarks appropriés (ces recommandations visent directement l'EU-ETS phase 3).

L'ensemble de ces recommandations sont explicitement repris par exemple dans les rapports au CDP de Lafarge, Holcim et CRH. Cemex fait mention des risques de compétitivité et renvoie au CSI en termes de recommandations. Heidelberger mentionne les dangers d'une baisse à terme des allocations gratuites pour la compétitivité de l'industrie européenne et renvoie aussi au CSI. Nous pouvons donc constater qu'il y a une grande homogénéité dans les positions des entreprises cimentières, homogénéité favorisée par la prééminence du CSI.

La voie de l’industrie chimique sur les questions climat - énergie est portée au niveau international par l'ICCA - International Council of Chemical Associations. La structure regroupe parmi ses membres la majorité des associations de chimistes tant au niveau local, que fédéral ou européen. Des membres dirigeants du CEFIC sont notamment en charge de la communication internationale de l'association. Toutes les entreprises étudiées sont intégrées dans le système de prise de position de l'association via la présence d'un ou de plusieurs représentants dans les comités ou les organes de direction de l'ICCA.

L'ICCA se positionne comme défenseur des mécanismes de marché comme outil économique favorisant la réduction des émissions de GES. Certaines pistes d'améliorations, biais et faiblesses réglementaires sont soulignées. La réglementation des émissions de GES doit se diriger vers un cadre international harmonisé en vue de réduire les risques de perte de compétitivité incarnée par les fuites carbone. L’incertitude concernant les cadres réglementaires 
auxquels seront soumis les chimistes doit être minimisée en vue de favoriser les prises de décision concernant des investissements. La spécificité de l'industrie chimique, au sens où elle fournit de nombreux secteurs, doit être prise en compte puisqu'elle offre un champ d'action majeur pour réduire les émissions de gaz à effet de serre. Un intérêt particulier est porté sur les marges d'amélioration de l'efficacité énergétique offertes par l'industrie chimique tant sur les procédés de production que sur les produits fournit à d'autre secteurs d'activité.

Ces positions se retrouvent dans les réponses apportées par les chimistes étudiés au CDP. Tous soutiennent une approche «marché global» pour réduire les émissions tout en soulignant l’importance de préserver la compétitivité du secteur tant que les réglementations ne seront que nationales. Notons une divergence d'appréciation entre DuPont et Rhodia sur les CDM (Clean Development Mechanism). Alors que Rhodia a beaucoup bénéficié de ces mécanismes de transferts entre pays émergents et pays industriels, DuPont a une position de principe hostile sur le sujet.

Cette comparaison sectorielle met en évidence que les deux secteurs sont également concernés par la mise en place de réglementations unilatérales débouchant sur des prix de $\mathrm{CO}_{2}$ différents d'une zone à l'autre. L'industrie cimentière apparaît cependant comme plus engagée pour la mise en place de dispositifs visant à limiter l'impact de ces prix différenciés sur la compétitivité (obtention d'allocations gratuites) alors que l'industrie chimique semble plutôt encourager l'harmonisation des politiques unilatérales.

\subsection{L'évolution globale des émissions de $\mathrm{CO}_{2}$ en lien avec l'évolution des portefeuilles d'activités (dimension D)}

Dans cette section, nous ne nous intéressons plus à la stratégie telle qu'elle est formulée en 2009 par les Présidents et à travers les objectifs affichés mais à la stratégie implicite telle qu'elle ressort des décisions passées au cours des dix dernières années. Pour des raisons d'accès aux 
données, cette analyse est menée sur un nombre réduit d'entreprises: trois entreprises pour chaque secteur.

Nous avons calculé l'évolution des émissions absolues de $\mathrm{CO}_{2}$ entre 1990 et 2009 et entre 2000 (ou à défaut 2003) et 2009, et mis ces évolutions en relation avec celle du chiffre d'affaires (cf. tableaux $5 \mathrm{a}$ et $5 \mathrm{~b}$ ). Nous avons également calculé l'évolution du chiffre d'affaires par activités (cf. tableaux 6a et 6b). L'examen des acquisitions et désinvestissements majeurs sur cette période, tels qu'ils figurent sur les sites internet/onglet histoire, permet d'interpréter le lien entre l'évolution du portefeuille d'activités et les enjeux du changement climatique.

\begin{tabular}{|c|c|c|c|}
\hline Entreprises & $\begin{array}{c}\text { Augmentation } \\
\mathbf{t} \mathbf{C O}_{2} \mathbf{2 0 0 9 / 1 9 9 0} \\
-\quad \text { en \% }\end{array}$ & $\begin{array}{c}\text { Augmentation } \\
\mathbf{t} \mathbf{C O}_{2} \mathbf{2 0 0 9 / 2 0 0 0} \text { ou } \\
\mathbf{2 0 0 9 / 2 0 0 3} \\
\mathbf{e n ~ \%}\end{array}$ & $\begin{array}{c}\text { Augmentation CA } \\
\mathbf{2 0 0 9 / 2 0 0 0} \text { en \% }\end{array}$ \\
\hline Lafarge & $22 \%$ & $30 \%$ & $30 \%$ \\
\hline Holcim & $41 \%$ & $32 \%$ & $56 \%$ \\
\hline Cemex & $-3 \%$ & & $159 \%$ \\
\hline
\end{tabular}

Tableau 5a - Evolution des émissions $\mathrm{CO}_{2}$ en lien avec les portefeuilles d'activités des entreprises cimentières

\begin{tabular}{|c|c|c|c|}
\hline Entreprises & $\begin{array}{c}\text { Augmentation t } \mathrm{CO}_{2} \\
2009 / 1990 \\
\text { en } \%\end{array}$ & $\begin{array}{c}\text { Augmentation t } \mathrm{CO}_{2} \\
2009 / 2000 \text { ou } 2009 / 2003 \\
\text { en } \%\end{array}$ & $\begin{array}{c}\text { Augmentation CA } \\
2009 / 2000 \text { en } \%\end{array}$ \\
\hline DuPont & $-78 \%$ & $-6 \%$ & $-7 \%$ \\
\hline Bayer & $-43 \%$ & $50 \%$ & $1 \%$ \\
\hline Rhodia & $-556 \%$ & $-80 \%$ & $-46 \%$ \\
\hline
\end{tabular}

Tableau 5b - Evolution des émissions $\mathrm{CO}_{2}$ en lien avec les portefeuilles d'activités des entreprises chimiques

(Sources : Calculs des auteurs à partir des rapports développement durable 2000-2009) 


\begin{tabular}{|c|c|c|c|c|c|c|}
\hline & \multicolumn{2}{|c|}{ Lafarge } & \multicolumn{2}{|c|}{ Holcim } & \multicolumn{2}{|c|}{ Cemex } \\
\hline Année & 2000 & 2009 & 2000 & 2009 & 2000 & 2009 \\
\hline Ciment & $36 \%$ & $60 \%$ & $65 \%$ & $65,34 \%$ & $76 \%$ & $46 \%$ \\
\hline $\begin{array}{l}\text { Granulats et } \\
\text { bétons }\end{array}$ & $31 \%$ & $32 \%$ & $23 \%$ & 28 & $23 \%$ & $51 \%$ \\
\hline Plâtre & $8 \%$ & $8 \%$ & \multirow{2}{*}{$12 \%$} & \multirow{2}{*}{$7 \%$} & \multirow{2}{*}{$1 \%$} & \multirow{2}{*}{$3 \%$} \\
\hline Autres & $25 \%$ & & & & & \\
\hline
\end{tabular}

\begin{tabular}{|c|c|c|c|}
\hline $\begin{array}{l}\text { Acquisitions } \\
\text { majeures }\end{array}$ & $\begin{array}{l}\text { - } 1994 \text { : développement en Chine } \\
\text {-1998: Redland (activités } \\
\text { toitures) } \\
\text {-2007: Orascom (Moyen-Orient), } \\
\text { Heracles Grèce) }\end{array}$ & $\begin{array}{l}\text {-2005 : développement en Inde } \\
\text {-2008 : développement en Chine } \\
\text {-2009 : ex-Rinker (Australie) }\end{array}$ & $\begin{array}{l}\text { - } 1992 \text { : les deux plus gros } \\
\text { cimentiers espagnols } \\
\text { - } 2000 \text { : Southdown (US) } \\
\text { - } 2005 \text { : RMC (Europe et } \\
\text { US) } \\
\text {-2007: Rinker (Australie) }\end{array}$ \\
\hline $\begin{array}{l}\text { Ventes } \\
\text { Majeures }\end{array}$ & $\begin{array}{l}\text { - } 2008 \text { : revente des activités de } \\
\text { Redland }\end{array}$ & & -2009 : Rinker (Australie) \\
\hline
\end{tabular}

\section{Tableau 6a - Evolution des portefeuilles d'activités des entreprises cimentières}

(Sources : Calculs des auteurs à partir des rapports annuels)

En termes de chiffre d'affaires, les trois entreprises du secteur cimentier ont toutes connu une très forte croissance. Cette croissance correspond à une globalisation de leurs activités avec un fort développement dans les pays émergents (Inde et Chine pour Holcim, Chine et Moyen Orient pour Lafarge, inversement rééquilibrage vers les pays développés pour Cemex avec l'acquisition de Southdown aux USA). Ces entreprises restent ancrées sur leur cœur de métier : ciment, béton prêt à l'emploi et granulats, le ciment jouant un rôle prépondérant. Au cours de la période, Lafarge qui s'était diversifié vers l'aval avec l'acquisition de Redland (activité toiture) en 1998, est revenu en arrière (même s'il conserve l'activité plâtre). Comme les caractéristiques de production sont relativement homogènes d'un pays à l'autre, cette focalisation sur le cœur de métier explique l'accroissement des émissions en valeur absolue pour Holcim et Lafarge. La baisse constatée pour Cemex correspond à l'accroissement du poids de l'activité granulats béton très complémentaire au ciment (acquisition de RMC en 2005 et de Rinker en 2007, cette dernière étant revendue en 2009 dans un contexte de crise financière). La baisse des émissions en intensité ( $\mathrm{tCO}_{2} /$ tciment) constatée au tableau $4 \mathrm{a}$ provient des améliorations régulières du processus de production (mix 
combustibles, efficacité énergétique, introduction d'ajouts au clinker) mais elle n’est pas suffisante pour changer substantiellement le ratio $\mathrm{tCO}_{2} / \mathrm{CA}$, ni a fortiori réduire les émissions totales. On comprend mieux pourquoi les entreprises du secteur cimentier n'ont pas affiché des objectifs de réduction d'émissions en valeur absolue, un tel objectif aurait été en contradiction avec leur stratégie de développement.

\begin{tabular}{|c|c|c|c|c|c|c|c|c|c|}
\hline & \multicolumn{3}{|c|}{ Rhodia } & \multicolumn{3}{|c|}{ Bayer } & \multicolumn{3}{|c|}{ Dupont } \\
\hline & Année & 2005 & 2009 & & 2000 & 2009 & & 2000 & 2009 \\
\hline \multirow{9}{*}{$\begin{array}{l}\text { Répartition } \\
\text { CA par } \\
\text { activité }\end{array}$} & $\begin{array}{l}\text { Agricult } \\
\text { ure }\end{array}$ & $20 \%$ & - & Agriculture & $11 \%$ & $22 \%$ & Agriculture & $14 \%$ & $32 \%$ \\
\hline & $\begin{array}{c}\text { Polyami } \\
\text { de }\end{array}$ & $38 \%$ & $37 \%$ & Santé & $32,30 \%$ & $51 \%$ & Santé & $5 \%$ & - \\
\hline & $\begin{array}{c}\text { Novecar } \\
\mathrm{e}\end{array}$ & $21 \%$ & $20 \%$ & $\begin{array}{c}\text { Matériaux \& } \\
\text { produits } \\
\text { chimiques }\end{array}$ & $46 \%$ & $23 \%$ & $\begin{array}{l}\text { Produits } \\
\text { Chimiques }\end{array}$ & & $19 \%$ \\
\hline & Silcea & $9 \%$ & $16 \%$ & Autres & $10 \%$ & $4 \%$ & Matériaux & $17 \%$ & $18 \%$ \\
\hline & Acetow & $9 \%$ & $14 \%$ & & - & - & Revêtement & $17 \%$ & $13 \%$ \\
\hline & $\begin{array}{l}\text { Energy } \\
\text { Services }\end{array}$ & $0 \%$ & $5 \%$ & & - & - & $\begin{array}{l}\text { Sécurité \& } \\
\text { Protection }\end{array}$ & $12 \%$ & $11 \%$ \\
\hline & $\begin{array}{c}\text { Eco } \\
\text { Services }\end{array}$ & $4 \%$ & $5 \%$ & & - & - & Électroniques & $11 \%$ & $7 \%$ \\
\hline & Autres & $-1 \%$ & $3 \%$ & & - & - & Textiles & $24 \%$ & - \\
\hline & & - & - & & & & Autres & $0,40 \%$ & - \\
\hline
\end{tabular}

\begin{tabular}{|c|c|c|c|}
\hline $\begin{array}{l}\text { Acquisitions } \\
\text { majeures }\end{array}$ & $\begin{array}{l}\text { - Portefeuille de type } \\
\text { conglomérat (Rhône } \\
\text { Poulenc) recentré sur la } \\
\text { chimie de spécialité, } \\
\text { industrie automobile, } \\
\text { électronique et électrique, } \\
\text { énergie etc... }\end{array}$ & $\begin{array}{l}\text { - Structuration sur Health Care, } \\
\text { Food/Seed, Material Science } \\
\text { - } 2006: \text { Icongenetics (semences) } \\
\text { - } 2006 \text { : Schering (contraception) } \\
\text { - } 2007 \text { : Stoneville Pedigreed Seed } \\
\text { ( } 2008 \text { : Direvo biotech (semences) } \\
\text { - } 2009: \text { Athenix (pesticides) }\end{array}$ & $\begin{array}{l}\text { - Evolution de la chimie fossile à } \\
\text { la chimie verte } \\
\text { - } 1997 \text { : Pioneer (biotech) }\end{array}$ \\
\hline $\begin{array}{l}\text { Ventes } \\
\text { Majeures }\end{array}$ & $\begin{array}{l}\text {-2004 : phosphates, } \\
\text { ingrédients alimentaires } \\
\text {-2005: CFC à DuPont } \\
\text { - } 2007 \text { : cession Nylstar } \\
\text { (fibres textiles } \\
\text { synthétiques) } \\
\text {-2008 : cession Rhodia } \\
\text { Organics }\end{array}$ & $\begin{array}{l}\text { - } 2000 \text { : Bayer Solar } \\
\text { - } 2005 \text { : Imagerie médicale } \\
\text { - } 2006 \text { : cellulose, silicone, polymères }\end{array}$ & $\begin{array}{l}\text {-1998 : Conoco (pétrole) } \\
\text { - } 2001 \text { : vente activité } \\
\text { pharmaceutique }\end{array}$ \\
\hline
\end{tabular}

\section{Tableau 6a - Evolution des portefeuilles d'activités des entreprises chimiques}

(Sources : Calculs des auteurs à partir des rapports annuels) 
La situation des entreprises dans le secteur chimique est assez contrastée par rapport au secteur cimentier. D’une manière générale, ces entreprises ont redéfini leur portefeuille d'activités. Sur la période 2000-2009, les trois entreprises sont plutôt en recul en termes de chiffres d'affaires (très nettement pour Rhodia). La baisse des émissions en valeur absolue pour DuPont est alignée avec cette baisse d'activité. Pour Bayer, le ratio $\mathrm{tCO}_{2} / \mathrm{CA}$ a d'abord monté puis commencé à descendre depuis 2007. Rhodia est la seule entreprise qui diminue fortement son ratio $\mathrm{tCO}_{2} / \mathrm{CA}$, du fait de son brûleur de $\mathrm{N}_{2} \mathrm{O}$ et de vente d'activités à forte intensité carbone comme la production de phosphates. Si ces trois entreprises affichent l'ambition de déconnecter leur croissance avec le niveau de leurs émissions absolues, seule Rhodia semble obtenir des résultats qui vont dans ce sens. Pour DuPont et Bayer, le passage de la chimie fossile à la chimie verte reste encore un pari sur l'avenir. Si on revient maintenant aux baisses d'émissions en valeur absolue sur l'ensemble de la période 2009/1990, au-delà des mouvements de portefeuille, l'importance de ces réductions s'explique aussi par l'introduction d'innovations majeures : par exemple l'élimination du CFC chez DuPont au début des années 1990 ou l'élimination du $\mathrm{N}_{2} \mathrm{O}$ chez Rhodia à partir de 1998. D’autres innovations sont annoncées notamment dans les procédés de production de chlorine par Bayer.

L'industrie chimique bénéficie de deux leviers d'action pour diminuer ses émissions. D’une part, elle est capable de modifier significativement son processus de production et d'en tirer parti sous forme de brevets et de transferts de technologie. D'autre part, l'industrie peut restructurer son portefeuille d'activités de façon majeure.

L'interprétation du changement de portefeuille n'est pas directement lisible pour les chimistes, contrairement au secteur cimentier. Nous prendrons en détail le cas des différentes entreprises. La restructuration est particulièrement nette dans les cas de Bayer et Dupont. Il est à noter que les démarches ci-après démontrent une capacité des entreprises à identifier en amont des secteurs à fort potentiels de croissance liés aux problématiques du changement climatique : 
agriculture, énergie, santé. En effet, les deux entreprises se positionnent fortement sur le domaine agricole qui représente une part non négligeable - 26.6\% - des réductions d'émission potentielles liées aux applications des produits de l'industrie chimique (ICCA - 2009). Entre 2000 et 2009, l'activité Crop science de Bayer a gagné quasi 11 points de part de chiffre d'affaire global de l'entreprise pour passer de $11 \%$ à $22 \%$ de celui-ci. Cette expansion s'accompagne d'une démarche d'acquisitions nombreuses et importantes d'entreprises spécialisées dans les semences et les pesticides. Sur la même période, Dupont voit la part de son activité agriculture et nutrition passée de $14 \%$ à $32 \%$.

La démarche de ces deux groupes est toutefois fort différente sur le second levier de croissance. Alors que Bayer investit massivement sur son activité santé qui représente maintenant plus de $50 \%$ de son chiffre d'affaire, Dupont avance sur le chemin des produits chimiques de performance majoritairement utilisés dans les domaines de l'efficacité énergétique et la production d'énergie renouvelable (panneaux solaires). Le choix de Bayer s'explique notamment par l'anticipation du développement de pathologies liées au changement climatique telles que la malaria, le paludisme, etc.

Rhodia a pris une toute autre voie. Suite aux restructurations multiples de ses activités entre 2000 et 2005, deux points sont à noter. Tout d'abord, les activités pharmaceutiques et agrochimiques regroupées au sein de Rhodia Organics - 20\% du chiffre d'affaire - ont été cédées en 2008. Le désinvestissement de Rhodia sur ce segment est particulièrement intéressant puisque cette décision va dans le sens inverse des deux autres entreprises étudiées. Le second aspect particulièrement notable de la stratégie de Rhodia est l'émergence d'activités directement liées aux émissions de $\mathrm{CO} 2$ ciblant ses clients et aux réglementations. Rhodia a su très tôt tirer parti des opportunités liées aux mécanismes de flexibilité liés au Protocole de Kyoto. Profitant de son innovation technologique sur les émissions de $\mathrm{N}_{2} \mathrm{O}$, Rhodia a pu développer des projets de réduction d'émissions donnant droit à des crédits de compensation nommés CER. Ces crédits sont autorisés comme outil de conformité pour les entreprises sous contrainte dans le cadre du 
système européen d'échange de quotas. Pour maximiser les retombées financières, Rhodia a constitué une joint venture avec la Société Générale (Orbeo). En parallèle, Rhodia, via la création de son activité Rhodia Energy Services, offre à ses clients des services de gestion de l'approvisionnement énergétique. Cette nouvelle branche représente d'ores et déjà 5\% du chiffre d'affaire du groupe. ${ }^{12}$ Il faudrait que l'industrie cimentière soit capable d'inventer un ciment sans clinker pour qu'on obtienne des résultats comparables. Ces décisions démontrent une volonté de repositionnement de son activité sur un secteur à forte croissance liée aux préoccupations environnementales : la production d'énergie à base de matériaux biologiques renouvelables.

\section{Discussion et conclusions}

Commençons par dégager une vision synthétique par secteur à partir de notre analyse empirique sur les 4 dimensions du tableau 1. Les entreprises du secteur cimentier que nous avons étudiées offrent une image assez homogène. Elles reconnaissent les enjeux associés au changement climatique dans leur vision stratégique (dimension A). Ces enjeux se traduisent à ce stade par des engagements en intensité en termes d'émissions (dimension B) tout en soulignant les dangers des réglementations unilatérales (type EU-ETS) sur la compétitivité des firmes (dimension C). Ces actions viennent s'inscrire à côté d'actions plus traditionnelles sur la préservation de l'environnement (pour l'exploitation des carrières) sans créer pour l'instant de nouvelles dynamiques stratégiques fortes. L'évolution de leur portefeuille d'activités s'explique largement par les enjeux de globalisation propres au secteur cimentier et pas par une volonté de tirer parti d'opportunités liées au changement climatique (dimension D). A ce stade, les enjeux liés au changement climatique ne semblent pas offrir d'axes de différenciation entre les entreprises du secteur : les objectifs de baisse d'émissions sont focalisés sur les émissions liées au processus de production du ciment et les résultats obtenus sont proches. On note cependant la

\footnotetext{
${ }^{12}$ DuPont poursuit aussi une telle stratégie via son activité de conseil Sécurité et Protection.
} 
mise en place de programmes internes pour aller vers des objectifs «marché » (produits solutions) associés à la thématique construction durable. Mais, même si les entreprises communiquent sur ces programmes (réalisations ponctuelles, partenariats), elles ne mentionnent en externe aucun objectif quantifié lié à ces programmes.

Cette image synthétique justifie à nos yeux pour ce secteur la validation des hypothèses 1 et 2 . Pour les entreprises cimentières, l'enjeu du $\mathrm{CO}_{2}$ est principalement centré sur le processus de production. Ces enjeux restent circonscrits et n'ont pas conduit pour l'instant à une remise en cause de la stratégie globale de l'entreprise. Compte tenu de l'homogénéité observée dans le secteur nous pouvons conclure au déterminisme des facteurs économiques sectoriels et dire que ces facteurs conduisent à des « figures imposées ».

Examinons maintenant la situation dans le secteur de la chimie. D’une manière générale, les entreprises étudiées ont tiré parti des enjeux liés au changement climatique pour reformuler leur vision stratégique (dimension A). A côté du $\mathrm{CO}_{2}$, certaines d'entre elles ont identifié des enjeux sociétaux globaux liés aux retombées du changement climatique sur l'agriculture et la santé. Les enjeux liés aux émissions proprement dites s'inscrivent dans une longue tradition liée à l'émanation de substances toxiques, substances maintenant marquées par des effets nuisibles à long terme. Les actions industrielles pour limiter les émissions correspondantes s'appuient sur les leviers classiques (efficacité énergétique) mais aussi sur des changements de procédé suite à des innovations majeures (bruleur de $\mathrm{N}_{2} \mathrm{O}$ chez Rhodia, et procédé de production de Chlorine chez Bayer). Les engagements associés apparaissent d'emblée hétérogènes (souvent en valeur absolue, correspondant à des dates d'engagement diverses, et surtout donnant lieu à des résultats très disparates d'une firme à l'autre). Quelques entreprises prennent des engagements « marché », mais elles restent minoritaires dans notre échantillon (dimension B). Les enjeux de compétitivité marquent aussi les positions vis-à-vis des réglementations unilatérales, la question des allocations 
gratuites est moins centrale, certaines entreprises voient une opportunité dans les CDM, d'autres y sont hostiles (dimension C). La différence avec l'industrie cimentière est manifeste quant à l'impact du changement climatique sur le portefeuille d'activités. Il est encore trop tôt pour parler d'une mutation de la chimie fossile vers la chimie verte mais notre analyse (dimension D) suggère qu'elle est en cours et qu'elle se traduit par des choix différents entre les firmes.

Dans ces conditions, il nous semble légitime de considérer que les hypothèses 1 et 2 sont validées en ce qui concerne le secteur de la chimie. L'enjeu du $\mathrm{CO}_{2}$ est plus diffus, il ne se limite pas aux enjeux strictement industriels, mais touchent l'ensemble de la chaine de valeur. La reformulation de la vision stratégique conduit ces entreprises à revoir leurs portefeuilles d'activités de manière volontariste pour exploiter de nouvelles opportunités. La diversité des stratégies observées est bien en phase avec l’idée de « figures libres ».

Même si nos résultats confirment le poids des facteurs économiques structurels dans les stratégies RSE des entreprises, il n'en reste pas moins vrai que notre analyse est limitée. Notre analyse est motivée par une étude de cas sur DuPont et Lafarge qui nous a conduits à proposer deux types de stratégies RSE liées aux enjeux du $\mathrm{CO}_{2}$. A ce stade, nous pouvons considérer que les différences observées sur DuPont et Lafarge se retrouvent dans une large mesure à un niveau sectoriel. Il conviendrait de valider cette observation de manière plus systématique en partant de variables vérifiables et recueillies sur un échantillon représentatif des secteurs concernés. Il conviendrait aussi d'étudier l'influence d'autres facteurs plus institutionnels (DiMaggio et Powell 1983), tels que le charisme de certains dirigeants, l'effet de mimétisme ou la pression exercée par les parties prenantes sur les choix stratégiques. Une telle analyse permettrait certainement d’identifier les raisons plus organisationnelles qui ont pu générer des différences entre entreprises au sein d'un même secteur : comme par exemple le leadership souvent cité de Lafarge au début des années 2000, ou celui de DuPont à la même période. 
En dépit de ses limites, cette étude nous incite à penser que la capacité des entreprises à innover face au changement climatique ne dépend pas uniquement de la capacité de l'entreprise à piloter ses «figures libres » et « figures imposées »(Aggeri et al. 2005), mais est fortement influencée par des facteurs structurels qui s'imposent à toutes les entreprises d'un même secteur. Ces résultats nuancent les propos avancés par les travaux sur la «Strategic CSR » (Porter et Kramer 2006, 2011) qui accordent une importance majeure aux actions menées par les entreprises pour transformer les contraintes environnementales en sources d'opportunité stratégique. En particulier, cette étude comparative montre que des contraintes structurelles fortes peuvent freiner les entreprises dans la transformation leur espace concurrentiel et social : une caractéristique essentielle des « figures libres ». Réciproquement, l'article démontre également que certains secteurs sont par nature plus favorables à des démarches proactives et innovantes. Bénéficiant de facteurs structurels plus favorables, les entreprises du secteur chimique se sont ainsi plus engagées dans le passage des «figures imposées » aux « figures libres », transformant la contrainte $\mathrm{CO}_{2}$ en une source d'innovation stratégique. Les facteurs structurels identifiés dans cet article - à savoir 1) la dépendance du processus de production vis à vis des ressources naturelles, 2) la capacité à jouer sur le portefeuille d'activités et le rôle induit pour la R\&D et 3) la structure du secteur aval - pourraient ainsi contribuer à la réflexion sur les politiques incitatives pour favoriser la croissance verte (Crifo et al. 2009) en fournissant des clés pour prendre en compte l'aspect sectoriel dans la réglementation. 


\section{Références}

Aggeri, F., Pezet, E., Abrassart, C., Acquier, A. (2005). Organiser le développement durable. Paris: Vuibert.

Arjaliès, D.-L., Ponssard, J.-P. (2010). A managerial perspective on the porter hypothesis: The case of co2 emissions. In Csr: From compliance to opportunity (Eds, Crifo, P., Ponssard, J.-P.). Palaiseau: Editions de l'Ecole Polytechnique.

Baumert, K. A., Herzog, T., Pershing, J. (2005). Navigating the numbers. Greenhouse gas data and international climate policy. World Resources Institutes 13.

Baumol, W. J., Panzar, J. C., Willing, R. D. (1982). Contestable markets and the theory of industry structure. New York: Harcourt Brace Jovanovitch.

Crifo, P., Debonneuil, M., Grandjean, A. (2009). Croissance verte: L'économie du futur. Rapport pour le Conseil économique pour le développement durable.

DiMaggio, P. J., Powell, W. W. (1983). The iron cage revisited: Institutional isomorphism and collective rationality in organizational fields. American Sociological Review 48 (2): 147-160.

Freeman, R. E. (1984). Strategic management: A stakeholder approach. Pitman Publishing.

Goodpaster, K. E. (1983). The concept of corporate responsibility. Journal of Business Ethics $2(1): 1-22$.

Heal, G. (2005). Corporate social responsibility: An economic and financial framework. The Geneva Papers 30 (3): 387-409.

Hommel, T., Godard, O. (2001). Contestation sociale et stratégies de développement industriel: Application du modèle de la gestion contestable à la production industrielle d'ogm. Les cahiers du laboratoire d'économétrie de l'Ecole Polytechnique Novembre 2001. 
Mc Kinsey Global Institute. (2008). The carbon productivity challenge: Curbing climate change and sustaining economic growth.

Morgensten, R. D., Pizer, W. A. (2007). Reality check : The nature and performance of voluntary environmental programs in the united states, europe and japan. RFF Press.

Nidumolu, R., Prahalad, C. K., Rangaswami. (2009). Why sustainability is now the key driver of innovation. Harvard Business Review 87: 57-64.

Porter, M. E. (1980). Competitve strategy: Techniques for analyzing industries and competitors. Free Press.

Porter, M. E., Kramer, M. R. (2006). Strategy and society - the link between competitive advantage and corporate social responsibility. Harvard Business Review 84 (12): 7892.

Porter, M. E., Kramer, M. R. (2011). Creating shared value: How to reinvent capitalism - and unleash a wave of innovation and growth. Harvard Business Review January-February 2011: 63-77.

Simons, R. (1995). Levers of control: How managers use innovative control systems to drive strategic renewal. Boston: Harvard Business School Press.

Stern, N. (Ed.) (2006). The stern review report: The economics of climate change. HM Treasury, London.

Vogel, D. (2005). The market for virtue - the potential and limits of corporate social responsibility. Brookings Institution Press.

Wood, D. J. (1991). Corporate social performance revisited. Academy of Management Review 16 (4): 691-718. 